\title{
Survey on wireless sensor networks: energy efficient optimization routing algorithms
}

\author{
V. Sivasankarareddy, G. Sundari \\ Department of Electronics and Communication Engineering, Sathyabama Institute of Science and Technology, India
}

\begin{tabular}{l}
\hline Article Info \\
\hline Article history: \\
Received Dec 13, 2019 \\
Revised Feb 14, 2020 \\
Accepted Feb 28, 2020 \\
\hline
\end{tabular}

\section{Keywords:}

Energy conservation Optimizing techniques Routing protocols Wireless sensor networks

\begin{abstract}
Wireless Sensor Network is extensively utilized in numerous places, such as protection surveillance. In Wireless Sensor Network, sensing unit networks are particular arbitrarily in addition to likewise in-network relying upon the technique is used to extend the network. As sensing unit nodes make use of strength from batteries for noticing the facts in addition to forwarding data, it uses the capability for those answers. The sizable troubles in cordless networks include power optimization, protection, directing, and project type. In this paper, current procedures in escaping power utilization of Wireless Sensor Network in addition to distinctive protocols and also Methods are researched. Additionally, destiny research have a look at on strength efficiency in Wireless Sensor Network putting forward new terms as well as targets for in addition examination is mentioned. Depiction of optimizing strategies like particle swarm optimization set of rules as well as ant swarm optimization Formula is already possible for lowering the electricity loss and complements the life of sensor community but those strategies take in greater time. This paper gives surveying extraordinary different optimization techniques below the multi-objective facet that takes region in tradeoffs. Information extracting in sensing unit networks is the technique of obtaining software-enabled plans in addition to patterns with gratifying accuracy from a constant, speedy, in addition to probable non-ended flow of facts streams from sensor networks. Various boundaries in preceding optimization Algorithms and suggesting a great deal better treatment through applying Data extracting Strategies for Wireless Sensing unit Networks is carried out.
\end{abstract}

Copyright $(0) 2020$ Institute of Advanced Engineering and Science. All rights reserved.

\section{Corresponding Author:}

V. Sivasankarareddy,

Department of Electronics and Communication Engineering,

Sathyabama Institute of Science and Technology,

Kamaraj Nagar, Semmancheri, Chennai, Tamil Nadu 600119, India.

Email: tejapav05@gmail.com

\section{INTRODUCTION}

Wireless Sensor Networks (WSNs) are sort of mini detectors, disbursed over a huge region to preserve an eye on adjustments inside the ecosystem. Gadgets connect to every other on demand with strategies created specifically for impartial interplay. The sensors are made using micro-electromechanical systems [1] that introduced approximately the advancement of constrained useful resource nodes. WSN locates its utility in diverse fields consisting of monitoring, surroundings, business tracking, fitness and health medical researches, and so forth [2]. Power sensors are used to send, reap, direct and additionally find out details that are shared with subsequent-door friends or a commonplace base station. Regular use of energy outcomes in degeneration of network connectivity due to interruptions, useless nodes or failing of connections and constrained courses to power overall performance as well as delay of community existence [3] Advice making use of strength issue is considered to attend to the strength use of gadgets with ultimate 
decision making. Several formulas have been counseled to control strength utilization, which ultimately intend to prolong the lifestyles of the grid.

To enlarge the lifestyles of wireless sensing unit networks, power tracking in keeping with sure performance requirements has changed into one of one of the maximum essential elements of the device. No count number precisely how precise the system fashion, the nodes will ultimately run out, if the only useful resource of strength is the battery. The strength collection organization wiring makes it feasible for effective conversion and additionally garage space of solar strength. Lately, springing up electricity collection era has provided a method for sustainable portable systems, that have theoretically unlimited runtime. As an instance, the primary model to hold the sensing unit with solar panels to acquire energy from the solar [4]. Collected electricity may be made use of certainly to reenergize the primary source of strength (for example, battery). Consequently, the device gives 2 source of power: (1) key battery while the gadget is implemented, and (2) consistent electricity series rate but now not usually hard.

In this paper, we are providing a quick survey on data extracting strategies for wireless sensing unit networks. A take a look at on clustering algorithms for WSNs is given in [5] The clustering strategies checked out in those documents distinctively perceive the structure and additionally control of the community instead of the exploration of details given in [6-7] and also offers a survey on records circulation type techniques, wherein the author has without a doubt explore trendy strategies for facts float. Unlike the above survey, this document specifically takes a take a look at algorithms and also strategies created for WSN laptop registries, which are not important for a sure class, evaluation, as well as conversation on specific domain names, likewise use extraordinary options. Additionally, this paper presents research work with distinct strength reliable optimization strategies. Even extra, the study method consists of testimonial of information mining strategies for WSNs.

\section{ENERGY EFFICIENT OPTIMIZATION TECHNIQUES}

In [8-17] is a multi-hop network without facilities wherein every node communicates with various other nodes simultaneously or circuitously via intermediate nodes. For that cause, all nodes in MANET must present themselves as cell routers that maintain a number of the directing methods had to find and preserve guides. Given that MANETs [18] are cordless, infrastructure-unfastened, self-prepared and fast launched networks, they're exceptionally in shape for programs that consist of specialized outdoor responsibilities, conversation in regions without $\mathrm{Wi}-\mathrm{Fi}$ infrastructure, emergency scenario and herbal. Tragedies and also naval operations have been included. Flow is an essential hassle in the mantra, specifically due to its dynamic and transferring nature. Particularly, surroundings-pleasant pressure transmitting can be amongst one of the maximum crucial fashion requirements for MANETs, because of the fact that mobile nodes can undergo the battery with restricted ability. Listed beneath we have in reality given survey on extraordinary energy powerful optimization directing protocols in MANET.

\subsection{Energy efficient optimization with load balancing}

Mobile Ad Hoc Networks (MANETs) a self-configured community of cell nodes connected via wireless links wherein each cell node acts as a number and router. With the growth and unfold of those gadgets in all elements of society, the want for such gadgets to communicate with transparency has end up increasingly important. MANET-compliant packages have strict QoS requirements, and to aid those satisfactory-of-provider parameters, MANET ought to have effective routing protocols [19-20]. Most interactive routing protocols inclusive of AODV [21] offer a single path for packet delivery. However, while a unmarried path fails, there is a lower in the overall performance of many QoS parameters. Providing a single backup route does not completely solve the problem, because the backup course may also fail. Additionally, imparting multiple backup paths may additionally purpose a couple of packets to glide at the community.

Manet got a super Wi-Fi conversation in a short period of time [18]. This is because of its flexibility, virtually low cost and scalability furnished by means of vibrant infrastructure. In this record, a layout based totally upon a line plan is proposed for MANETs to assess general efficiency metrics. The node may be concept about as a server to offer applications that reach the vacation spot node. Packets get to every node in a safe group, satisfy favorable analytical distributions and take a look at a sure design. M/ G/ 1: The MANET or/ FIFO variation has been drafted, which assesses a few common ready time (AWT), and reaction instances (RT), Queuing hold-up (QD).

Wireless sensor network applications that help many fields have gained critical recognition and interest from researchers these days. WSN packages are designed to support real-time and scalable monitoring of the sharing environment [22]. The prevailing attitudes at WSN [23] include energy optimization, routing, obstacle identity, security, etc. These constraints require priority improvement to meet the needs of the implementing environment. Many study methods provide answers to each of the factors that 
affect WSN. We intend to study the techniques of improving electricity in WSN with different concerns. This document focuses on examining past improvement strategies under a multi-objective position leading to compensation. We exclude known optimization methods [24-32] and examine great, accurate measurement improvements based on link disruption, load balancing, and distance.

In [33] dynamic topology and additionally more than one input nature. This makes it extremely challenging to offer QoS parameters in those networks. Among the predominant elements for the low fine of service in MANET is the lack of packets because of the sudden breakdown of hyperlinks. Consequently, it's far essential to provide a transmitting protocol that remains to give backup techniques accurately when the grasp path falls brief. In addition, multimedia streaming has grown to be a necessity nowadays with packages in videoconferencing, essential image transmission, and emergency state of affairs as well as many greater. All these programs have to be certified with QoS, which makes positive specific efficiency, exact package value price, and so on, in addition to this may nice be ensured by way of an effective transmitting protocol. Multimedia streaming in MANET need to also perform in scattered as well as dense MANET.

\subsection{Distance based energy optimization techniques}

In [35] based totally green electricity goal tracking formula, wherein we discuss network safety for sensor nodes and endorse a fixed of regulations for strength effectiveness, goal monitoring and also efficiency renovation. In the WSN neighborhood to reap high energy overall performance, we ought to avoid long-distance communiqué. We recognize the optimization of the made use of show, with the most useful display of the sensor, and also at the same time as we check the objective of boosting energy usage, we can make use of target interest data. To extract the preceding records, particle removal (PF) may be thought approximately. Thinking about the progressed strength within the sensor application and also goal manage in WSN. This advocated set of rules makes a specialty of same maintenance to cover power performance and additionally dynamic electric energy management. The optimization firm is dispatched to many heads of the block.

The recommended green ant-primarily based lots harmonizing (EBAR) guidance set of rules supplied, by means of Xinlu Li et [36] the EBAR adopts a fixed of arbitrary course detection pointers and a scent cutting-edge direction alternative system to aid the energy usage of sensing unit nodes. It makes use of an green overall performance substitution components based on greedy energy rate scale. Anticipated to decorate the creation of the session. Ultimately, to be able to limit electricity intake due to public administration expenses, EBAR utilizes a fully opportunistic strength-based totally transmission system. We resemble WSN in numerous public provider attitudes to review EBAR at the same time as approximating trendy efficiency metrics at the side of electricity utilization, electric energy performance and the predicted helpful existence of the vicinity.

In [37] Recommended to send solid facts among sensing unit nodes to the receiving node, we have cautioned community inscribing based totally upon XOR, suggesting PLUVIAL layer. Unlike the preceding tactics within the literary works, we offer random sensing unit nodes in vicinity explained previously. Each delicate node is attached to at least one in addition to the best sink node, relying upon the Euclidean variety in addition to the variety of jumps in between them that is carried out with the assistance of Nature's Particle Swarm Enhancement (PSO) method.

Recommended modern-day technology to Avoidance Mobility-Assisted Localization the usage of Flock Intelligence Company (SIO) [38] of the site that helps him browse to live clear of limitations in cordless sensor networks. In many wireless sensing unit community (WSN) programs, the node region is wanted to spot the occasion being monitored as quickly as it takes place. Site-assisted navigating has turn out to be an efficient technique to settlement website. It improves course by using making put together for a mobile node with vicinity popularity, known as Mobile Support (MA). The venture for MA is to go across the area of passion (community) in a manner that lessens on-web page mistake even by optimizing a a hit nearby node series. To streamline, several management plan models count on the MA to have good enough toughness and time, which the community region is barrier-free.

\section{VARIOUS DATA MINING TECHNIQUES IN WIRELESS SENSOR NETWORKS CLUSTERING}

Clustering is an unsupervised study, in which the specific statistics are labeled right into parts to make certain that each subset stands for a team with unique homes. It is a useful approach that has honestly been taken under consideration in particular for applications that need scalability for a selection of sensor nodes. Gathering additionally sustains accumulating of realities in an try and summarize most of the people information transmitted. In modern literary works, troubles related to aggregation are resolved by using accumulating of nodes or collecting data. Just currently, masses of node gathering formulation have been created for WSN. These gathering techniques [39] vary notably of their objectives depending upon node 
release, startup structures, oppressed neighborhood framework, collection head capabilities (CH), and additionally network operation version. Although the set of nodes might be linked to aggregation of details, for example, presented the similarity of bordering node information, a number of the well-known node collecting algorithms that break up the nodes proper into a few tiny companies and additionally define a block header for each enterprise you do now do not utilize mining stats methods directly. In this check, we just consciousness on placing together reality-finding techniques to remove environmental information and additionally find data web hyperlinks in some nodes. Figure 1 recommends a synthesis of information generally made use of inside the widespread mining technique.

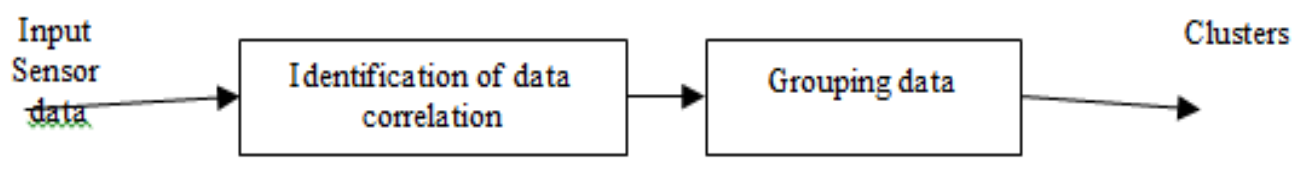

Figure 1. Data clustering for sensor networks

\subsection{Centralized graph-based energy-efficient data collection (EEDC)}

EEDC in [40-43] stands organized for a set of meeting hints that team nodes into groups in order that taxpayers have comparable sensor analyses, and as a result, the protocol accumulations the hook up with a recognition of the identified sensations. EEDC is a central method wherein the basin compares the information of a contract incredibly of version diagnosed by using the purchaser. EEDC creates a crew intro machine as a hassle for enthusiasts of disturbance by means of developing a graph GSo that every sensor node is a crest inside the graph. A facet $(\mathrm{u}, \mathrm{V})$ attracts if the evaluation step in between the vertex $\mathrm{u}$, and the vertex $\mathrm{V}$ is less than or identical to the described mass set.

These paintings adjusted techniques based totally on K-Means, hierarchical and pragmatic connection. The okay-mean series of guidelines takes the enter specification, good enough, and additionally creates a set of $\mathrm{n}$ objects in groups to ensure that the similarity inside the ensuing set is immoderate; despite the fact that, the resemblance among the teams is decreased. Team resemblance is determined with recognize to the implicit value of the aspects inside the team.

\subsection{Clustered aggregation algorithm (CAG)}

CAG in [44-48] set of rules is a fixed of regulations that combine nodes paperwork that reveal equal values inside a provided restriction (spatial correlation) in addition to stay unchanged as long as the sensor values continue to be within a restrict for the duration of the years. By grouping the agreement into equal values, the beneficial CAG sends loads more via have a look at in step with the established order. When border values are traded, the relevant sensing unit nodes will communicate with neighboring nodes related with diverse organizations to switch in among group subscriptions. CAG [49-50] ensures that of entirety result drops in the restriction of errors tolerance by the person. Teams are evolved for the duration of the dissemination of appointments to the neighborhood (exam phase), in which the group's organization unearths comparable values. Next off, CAG enters the reaction segment wherein the fundamental complete price in keeping with the mass is dispatched through the collection tree. CAG is a hard and fast of setting up policies with loss that exchanges the precision of the results of wonderful durability, garage, computation as well as provision of spoken trade [51]. Comparison on few data mining techniques as shown in Table 1.

Table 1. Comparison on few data mining techniques

\begin{tabular}{|c|c|c|c|}
\hline $\begin{array}{l}\text { Data mining } \\
\text { technique }\end{array}$ & Merits & Demerits & Applications \\
\hline Clustering & $\begin{array}{c}\text { Increased resource availability, } \\
\text { strategic resource usage, increased } \\
\text { performance, greater scalability and } \\
\text { simplified management }\end{array}$ & $\begin{array}{l}\text { With global cluster- it didn't work well; } \\
\text { different initial partitions can result in } \\
\text { different final clusters }\end{array}$ & $\begin{array}{l}\text { Market research, pattern } \\
\text { recognition, data analysis, } \\
\text { and image processing }\end{array}$ \\
\hline EEDC & $\begin{array}{l}\text { Network lifetime, number of nodes, } \\
\text { routing trees size }\end{array}$ & $\begin{array}{l}\text { Maximization of network, heterogeneous } \\
\text { communication capabilities are not dealt, no } \\
\text { measures to cope with unexpected delay }\end{array}$ & $\begin{array}{l}\text { Data fusion, multi-hop } \\
\text { communication and many to } \\
\text { one traffic pattern. }\end{array}$ \\
\hline CAG & Faster for low dimensional data & $\begin{array}{c}\text { Can't handle data of different sizes and } \\
\text { densities, restricted to data which has notion } \\
\text { of a centroid }\end{array}$ & $\begin{array}{l}\text { Identifying cancerous data, } \\
\text { search engines, academics, } \\
\text { drug activity prediction }\end{array}$ \\
\hline
\end{tabular}




\section{CHALLENGES IN PREVIOUS TECHNIQUES}

Challenges for the following reasons, traditional information mining strategies for handling sensor records in WSN are challenging. In [52]. Sensor nodes are constraints on resources useful in power, memory, bandwidth, and computing power statements. The most important task you face with the help of WSN's information mining techniques is to meet the needs of mining accuracy while keeping the consumption of aid in WSN to a minimum.

In [53] WSN, environment statistics are geographically dispersed, inputs are continuously arriving and recent record items can change results based on historical statistics. Most data mining techniques that analyze offline statistics are not compatible with handling distributed trading statistics. Therefore, the data mining technology company is a form of distribution method of data transmission over the Internet.

Data Transformation in [54] because sensor nodes are limited in bandwidth terms, it is not possible to reformulate real facts in society. Transforming knowledge is a vital problem. After the form and styles are extracted regionally from WSN records, the output is transferred to the lower station. Customization of the record extraction approach is how to efficiently create existing records and patterns in the community for transfer. To address these challenges, researchers have modified the conventional data mining techniques and also proposed new data mining algorithms to handle the data generated from sensor networks.

\section{COMPARISION OF OPTIMIZATION ROUTING ALGORITHMS}

\subsection{Ant colony optimization (ACO) algorithm}

The proposed ant colony improvement algorithms for wi-fi sensor networks. The ant colony optimization algorithm (ACO) [55-58] is widely used to improve the course in WSN. The standard set of Ant-Net rules for the cycle, which only take into account the main direction and reduce electricity consumption in large quantities, recommends this leads to a wide range of ants on the shortest route, and leads to very fast power consumption in the best path and death of the node. Therefore, the entire network lifecycle is reduced.

IQ Squadron is a new discipline that participates in the study of self-regulation techniques, both in nature and in artificial structures. Improving the ant colony [59-61] is an example of a swarm of intelligence. Ants are biologically optimized with the help of ant behavior. One ant is easy in nature and has a limited amount of memory, so it may seem easy. Not one ant will be sensitive enough, but ant colonies represent colony complex behavior by identifying clever solutions to problems as an example of trying to find the shortest route from the nest to the food source. An ant colony may be able to do the following; plan, locate and improve food routes.

\subsection{Fuzzy logic ant colony optimization (FACO) algorithm}

Scattered machine is a very important tool because we are able to integrate the human sort of thinking to do systems well. This research have a look at utilized doubtful reasoning to include key parameters so one can decide the maximum useful extraordinary deal with for a package dispatched out from the deliver sensor node to eviction. Great breeding requirements are made use of to assess the most remarkable node price using diffusion system input parameters, sensor network electricity in addition to internet traffic, and also final results is the very best node price. The network controller is the fish tank node. Mileage is the simplest connecting roadway for each and every sensor node. Works as a portal (excursion spot) for this WSN to bring together all community information. The sink node is the only that assesses the cost of every sensing unit node to the gate, masses the primary course of every sensing unit node and reveals the direction time table to the sensor nodes. The sink node is the only that makes use of a mystical rational controller to set up the fee of each sensing unit node to attain the sink node. Seek recommendation from all sensing unit nodes to ship details concerning your electric energy on-line popularity and also vacationer standing. The relationship rate is reviewed via the Fuzzy Reasoning Controller (FLC) [62-64] inputs that stand for the transportation electricity and visitors. An ant swarm optimization formulation may be a truly standard set of speculative search policies. It is inside the legance of the swarm knowledge and also is stimulated by way of the ants' technique that searches for the shortest direction in among the nest in addition to meals deliver. In this survey, an ant colony is used to calculate the shortest cycle, that's an top notch manner to apply it after the thick machine determines the only node expenses. As soon because the cost of all applicable hyperlinks is calculated on the supply node, the path can be recognized using the quickest scheduled cycle to set the maximum deal with. As quickly as one of the most right direction is computed, the sink node is the most effective one that famous the directing program for every sensor node to send out records. 


\subsection{Particle swarm optimization (PSO) algorithm}

It is inspired by flocking behavior of birds to solve continuous problem. The PSO [65-67] is a population based stochastic approach for solving continuous and discrete optimization problem. In PSO, simple software agents called particles move in the search space of an optimized problem. In the environment the nodes are to deployed, and by using the Particle swarm optimization technique [68] the cluster and cluster head are formulated and the residual energy of the nodes reduced. By the gravitational search algorithm the shortest route path is determined. In the performance analysis-the residual node reduction, load balancing ratio, total energy consumed, packet delivery ratio and network lifetime would be calculated. The PSO allows higher throughput i.e. solving more complex problems in the same time span and higher accuracy.

\subsection{Distributed joint optimization routing algorithm based on AHP (DORAHP)}

The suggested in [69] commonplace optimization model, primarily based upon AHP (Analytic Power Structure Refine), the staying energy of the node, the node space to BS (Base station) in addition to the node certificates are three essential choice requirements. Initially, AHP utilizes a regular matrix to approximate the weights of political election necessities. Second, AHP determines the charge of fellows or girls to each bordering node. Afterwards, all adjacent nodes are diagnosed in keeping with the overall weights of the choice requirements. Finally, the encompassing node with the most complete weight involves be the following jumping node of the transmitter.

AHP in [70] is a multi-preferred modeling era that splits a complex trouble into some simple subtroubles as well as establishes a 3-layer hierarchical shape. The main layer suggests the feature of the selection; the second one layer consists of the selection standards, while the bottom layer consists of several alternatives to the option. According to the body, DORAHP [71-73] develops a hierarchical structure as acquired Number 2. Its pinnacle layer determines the entire weights of nearby nodes, and also its twodimensional layer carries 3 choice criteria (i.e. Ordinary node resistance, node space to BS, and additionally node degree). The backside layer affords a collection of adjacent nodes, in which the node is picked as the next transition node. The hierarchical framework of DORAHP as shown in Figure 2.

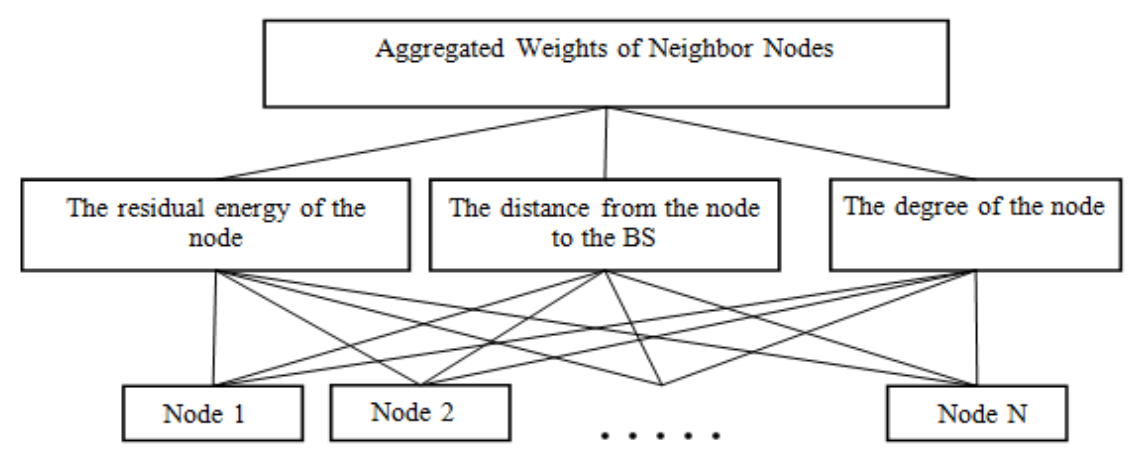

Figure 2. The hierarchical framework of DORAHP

\subsection{Energy-efficient routing algorithm based on the radar chain (EERC)}

An EERC in [74-78] has been proposed to increase network life. Based on the selection criteria (i.e., the remaining power of the node, the distance from the node to BS and the distance between the node and its adjacent nodes), this guidance system creates a complete and consistent AHP-based model for tuning the burden of the rules of each decision. The decision criteria for our proposed scheme conform to the EERC standards, but the gap between the node and its adjacent nodes is replaced by the EERC with the node score, which is the geographic characteristic of the node that affects the frequency of the node router. In addition, the residual force of the node and the distance between the node and BS represent the property of node resistance and distance property of the node, respectively.

\subsection{Low energy adaptive clustering hierarchy (LEACH) algorithm}

The LEACH in [79-81] set of policies gives the balancing power optimization throughout sensing unit area through random rotation $\mathrm{CHs}$ (collection heads). The $\mathrm{CH}$ option with the aid of the sum of power of node should be above threshold stage. After selection of $\mathrm{CH}$, the new node will broadcast its place to all bordering nodes then the nodes their information transmission using the $\mathrm{CH}$ to decrease crash based absolutely on TDMA (Time Department Several Access). In TDMA, the ports for every surrounding nodes permits energy intake low as nodes switch off their radio transmission whilst they might be in still 2nd. 


\subsection{Geographical energy aware routing (GEAR) algorithm}

In GEAR in [82-84] algorithm, the GPS is handled to the splitting up of nodes inside the sensor network. The base station is positioned at one point some distance from the observed network, front node is repaired at the primary factor of picked up community in addition to a limit distance price is specific. If the gotten distance is smaller than the restriction worth then the information transmission occurs directly. If the received distance is greater than the brink value after that inside the sensor community $\mathrm{CH}$ is chosen for information transmission. The political election of $\mathrm{CH}$ is based totally upon the possibility function and the residual strength of nodes. For this purpose the higher consequences may want to seem in phrases of network existence time, electricity intake in addition to programs transmission to the sink node or base station.

\subsection{Genetic algorithm (GA)}

The genetic algorithm [85-87] solves the electricity optimization difficulty. In GA, the major ideas are reproduction, fitness and fitness, crossover as well as mutation. The hereditary algorithms can be put on domains about which there wishes information or the dimension and/or complexity are too steeply-priced, or onerous, for analytic solution. In GA, the base station is looked after and it looks after more variety of sensor nodes in a network. Comparison between various routing protocols as shown in Table 2.

Table 2. Comparison between various routing protocols

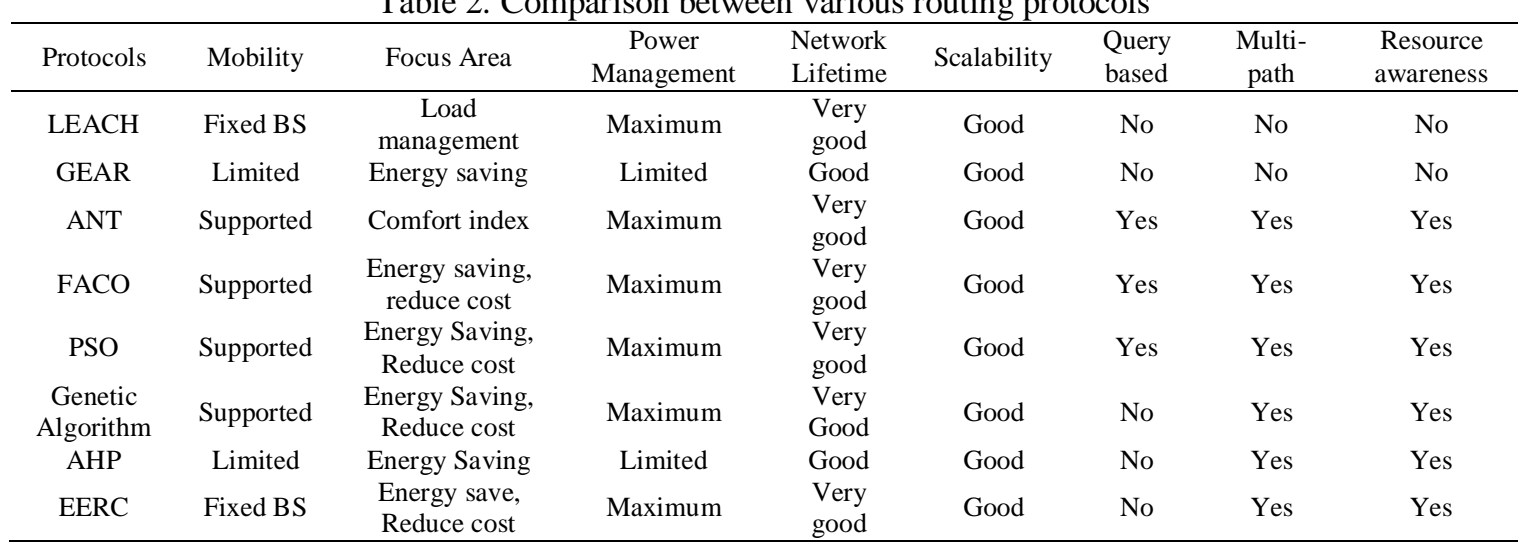

\section{CONCLUSION}

Wireless Sensor Networks applications will grow more popular with the improvement of sensor technology, and sensor data will become more information-rich. This paper studies various energy optimization methods with several detentions in WSNs. We examined the existing techniques on energy conservation and optimization with multiple approaches. The proposed strategies intend to reduce the adjustments in optimization when analyzing multi-objective metrics. Given various results between existed optimization algorithms in throughput and network lifetime. In this paper, first we have gone through a comprehensive survey of routing techniques in wireless sensor networks and observed various challenges in previous data mining techniques for WSNs. One of the significant criteria of wireless sensor network is energy efficiency. Focused on the energy problem, researchers design a few algorithms based on clustering in the present era but lack of proper $\mathrm{CH}$ election is prone to errors as marginal nodes are left out of recently established clusters. To fill these research gaps, further contribution we proposed better Data mining techniques for Wireless Sensor Network for energy efficient.

\section{REFERENCES}

[1] Tsai, Hsiung-Kuang, and Wen-Jian Lin. "Micro electro mechanical system display cell and method for fabricating thereof." U.S. Patent 6,882,461, issued April 19, 2005.

[2] Gaber, Mohamed Medhat, Arkady Zaslavsky, and Shonali Krishnaswamy. "A survey of classification methods in data streams." In Data streams, pp. 39-59. Springer, Boston, MA, 2007.

[3] Rao, Meena, and Neeta Singh. "Quality of service enhancement in MANETs with an efficient routing algorithm." In 2014 IEEE international advance computing conference (IACC), pp. 381-384. IEEE, 2014.

[4] Hu, Chenming, and Richard M. White. "Solar cells: from basic to advanced systems.".

[5] Boyinbode, Olutayo, Hanh Le, Audrey Mbogho, Makoto Takizawa, and Ravi Poliah. "A survey on clustering algorithms for wireless sensor networks." In 2010 13th International conference on network-based information systems, pp. 358-364. IEEE, 2010. 
[6] Ali, Ahmad, Yu Ming, Sagnik Chakraborty, and Saima Iram. "A comprehensive survey on real-time applications of WSN," Future internet 9, no. 4:77. 2017.

[7] Choi, Cheon Won. "Basic MAC Scheme for RF Energy Harvesting Wireless Sensor Networks: Throughput Analysis and Optimization." Sensors 19, no. 8: 1822. 2019.

[8] Raja, L., and S. Santhosh Baboo. "An overview of MANET: Applications, attacks and challenges." Int. J. of Comp. Sci. Mobile Comput. (IJCSMC) 3: 408-417. 2014.

[9] Marti, Sergio, Thomas J. Giuli, Kevin Lai, and Mary Baker. "Mitigating routing misbehavior in mobile ad hoc networks." In Proceedings of the 6th annual international conference on Mobile computing and networking, pp. 255-265. ACM, 2000.

[10] Parimala, R. "Mobile Ad Hoc Network Using Routing Algorithm Based on location Information by Mesh Topology." Journal of Mobile Computing, Communications \& Mobile Networks 5, no. 3: 11-14. 2019.

[11] Kumar, Sandeep, and Col Dr Suresh Kumar. "Study of MANET: Characteristics, Challenges, Application, Routing Protocol and Security Attacks." International Journal of $R \& D$ in Engineering Science and Management (ISSN 2393-865X) 2, no. 5: 266-274. 2015.

[12] BR, Arun Kumar, Lokanatha C. Reddy, and Prakash S. Hiremath. "Mobile Ad Hoc Networks: Issues, Research Trends and Experiments." (2008).

[13] Rishiwal, Vinay, Shekhar Verma, and S. K. Bajpai. "QoS based power aware routing in MANETs." International Journal of Computer Theory and Engineering 1, no. 1: 49. 2009.

[14] Gill, Anju, and Chander Diwaker. "Comparative Analysis of routing in MANET." International Journal of Advanced Research in Computer Science and Software Engineering 2, no. 7 (2012).

[15] Chin Kwan-Wu, John Judge, Aidan Williams, and Roger Kermode. "Implementation experience with MANET routing protocols." ACM SIGCOMM Computer Communication Review 32, no. 5: 49-59. 2002.

[16] Kurkowski, Stuart, Tracy Camp, and Michael Colagrosso. "Manet simulation studies: The current state and new simulation tools." Mobile Computing and Communications Review 9, no. 4: 50-61. 2005.

[17] Patel, Anal, Nimisha Patel, and Rajan Patel. "Defending against wormhole attack in MANET." In 2015 Fifth International Conference on Communication Systems and Network Technologies, pp. 674-678. IEEE, 2015.

[18] Ali, Aleem, and Neeta Singh. "Queueing approach based MANET performance analysis." In 2017 7th International Conference on Cloud Computing, Data Science \& Engineering-Confluence, pp. 422-424. IEEE, 2017.

[19] Manikannu, Jayekumar, and V. Nagarajan. "A survey of energy efficient routing and optimization techniques in wireless sensor networks." In 2017 International Conference on Communication and Signal Processing (ICCSP), pp. 2075-2080. IEEE, 2017.

[20] Rao, Meena, and Neeta Singh. "Performance analysis of AODV nthBR protocol for multimedia transmission under different traffic conditions for sparse and densely populated MANETs." In 2015 International Conference on Green Computing and Internet of Things (ICGCIoT), pp. 1010-1015. IEEE, 2015.

[21] Chakeres, Ian D., and Elizabeth M. Belding-Royer. "AODV routing protocol implementation design." In 24th International Conference on Distributed Computing Systems Workshops, 2004. Proceedings, pp. 698-703. IEEE, 2004.

[22] Ch. Rami Reddy, and K. Harinadha Reddy. "A New Passive Islanding Detection Technique for Integrated Distributed Generation System Using Rate of Change of Regulator Voltage over Reactive Power at Balanced Islanding." Journal of Electrical Engineering \& Technology 14.2: 527-534. 2019.

[23] Sharma, Mridula, Fayez Gebali, Haytham Elmiligi, and Musfiq Rahman. "Network Security Evaluation Scheme for WSN in Cyber-physical Systems." In 2018 IEEE 9th Annual Information Technology, Electronics and Mobile Communication Conference (IEMCON), pp. 1145-1151. IEEE, 2018.

[24] Kulkarni, Raghavendra V., and Ganesh Kumar Venayagamoorthy. "Particle swarm optimization in wireless-sensor networks: A brief survey." IEEE Transactions on Systems, Man, and Cybernetics, Part C (Applications and Reviews) 41, no. 2: 262-267. 2010.

[25] Shankar, T., S. Shanmugavel, and A. Karthikeyan. "Modified harmony search algorithm for energy optimization in WSN." Journal of International Review on Computers and Software 8, no. 6: 1469-1475. 2013.

[26] Shamsan Saleh, Ahmed M., Borhanuddin Mohd Ali, Mohd Fadlee A. Rasid, and Alyani Ismail. "A survey on energy awareness mechanisms in routing protocols for wireless sensor networks using optimization methods." Transactions on Emerging Telecommunications Technologies 25, no. 12: 1184-1207. 2014.

[27] Li, Yuzeng, Jianjun Xing, Qiaohe Yang, and Huichang Shi. "Localization research based on improved simulated annealing algorithm in WSN." In 2009 5th International Conference on Wireless Communications, Networking and Mobile Computing, pp. 1-4. IEEE, 2009.

[28] Wang, Jin, Yiquan Cao, Bin Li, Hye-jin Kim, and Sungyoung Lee. "Particle swarm optimization based clustering algorithm with mobile sink for WSNs." Future Generation Computer Systems 76: 452-457. 2017.

[29] Raghunathan, Vijay, Saurabh Ganeriwal, and Mani Srivastava. "Emerging techniques for long lived wireless sensor networks." IEEE Communications Magazine 44, no. 4: 108-114. 2006.

[30] Lin, Ying, Jun Zhang, Henry Shu-Hung Chung, Wai Hung Ip, Yun Li, and Yu-Hui Shi. "An ant colony optimization approach for maximizing the lifetime of heterogeneous wireless sensor networks." IEEE Transactions on Systems, Man, and Cybernetics, Part C (Applications and Reviews) 42, no. 3: 408-420. 2011

[31] Nayyar, Anand, and Rajeshwar Singh. "A comprehensive review of ant colony optimization (ACO) based energyefficient routing protocols for wireless sensor networks." International Journal of Wireless Networks and Broadband Technologies (IJWNBT) 3, no. 3: 33-55. 2014.

[32] Arjunan, Sariga, and Pothula Sujatha. "Lifetime maximization of wireless sensor network using fuzzy based unequal clustering and ACO based routing hybrid protocol." Applied Intelligence 48, no. 8: 2229-2246. 2018. 
[33] Rao, Meena, and Neeta Singh. "Simulation of various QoS parameters in a high density Manet set-up using AODV nthBR protocol for multimedia transmission, data transmission and under congestion scenario." ICTACT J Commun Technol 6, no. vol 3: 1155-1159. 2015.

[34] Deepa, S., S. Kamal Raj, and K. P. Sridhar. "Development of mobile adhoc network using leader Selection algorithm." International Journal of Engineering \& Technology 7, no. 2: 6-11. 2018.

[35] Tikhe, Khushbu, and Niyati Sohni. "PF-RBF based energy efficient target tracking routing algorithm for WSN." In 2016 International Conference on Automatic Control and Dynamic Optimization Techniques (ICACDOT), pp. 532-537. IEEE, 2016.

[36] Li, Xinlu, Brian Keegan, Fredrick Mtenzi, Thomas Weise, and Ming Tan. "Energy-Efficient Load Balancing Ant Based Routing Algorithm for Wireless Sensor Networks." IEEE Access. 7: 113182-113196. 2019.

[37] Meena, Yogesh Kumar, Amit Singh, and Anup Singh Chandel. "Distributed Multi-Tier Energy-Efficient Clustering." International Journal of Computer Theory and Engineering 4, no. 1: 1. 2012.

[38] Gautam, Monika, and Vikas Sejwar. "A brief review of WSN energy and routing protocols." In 2017 International Conference on I-SMAC (IoT in Social, Mobile, Analytics and Cloud)(I-SMAC), pp. 849-855. IEEE, 2017.

[39] Chao, Chih-Min, and Tzu-Ying Hsiao. "Design of structure-free and energy-balanced data aggregation in wireless sensor networks." Journal of Network and Computer Applications 37: 229-239. 2014.

[40] Villas, Leandro A., Azzedine Boukerche, Horacio ABF De Oliveira, Regina B. De Araujo, and Antonio AF Loureiro. "A spatial correlation aware algorithm to perform efficient data collection in wireless sensor networks." Ad Hoc Networks 12: 69-85. 2014.

[41] Anastasi, Giuseppe, Marco Conti, and Mario Di Francesco. "Reliable and energy-efficient data collection in sparse sensor networks with mobile elements." Performance Evaluation 66, no. 12: 791-810. 2009.

[42] Nguyen, Minh Tuan, and Nazanin Rahnavard. "Cluster-based energy-efficient data collection in wireless sensor networks utilizing compressive sensing." In MILCOM 2013-2013 IEEE Military Communications Conference, pp. 1708-1713. IEEE, 2013.

[43] Nguyen, Minh Tuan, Keith A. Teague, and Nazanin Rahnavard. "CCS: Energy-efficient data collection in clustered wireless sensor networks utilizing block-wise compressive sensing." Computer Networks 106: 171-185. 2016.

[44] Tang, Jine, ZhangBing Zhou, Jianwei Niu, and Qun Wang. "An energy efficient hierarchical clustering index tree for facilitating time-correlated region queries in the Internet of Things." Journal of Network and Computer Applications 40 (2014): 1-11.

[45] Yuea, Jun, Weiming Zhang, Weidong Xiao, Daquan Tang, and Jiuyang Tang. "Energy efficient and balanced clusterbased data aggregation algorithm for wireless sensor networks." Procedia Engineering 29: 2009-2015. 2012.

[46] Ma, Yajie, Yike Guo, Xiangchuan Tian, and Moustafa Ghanem. "Distributed clustering-based aggregation algorithm for spatial correlated sensor networks." IEEE Sensors Journal 11, no. 3: 641-648. 2010.

[47] Abdulsalam, H. M., and Kamel LK W-LEACH. "Weighted Low Energy Adaptive Clustering Hierarchy aggregation algorithm for data streams in wireless sensor networks." In Proceedings of IEEE International Conference on Data Mining Workshops (ICDMW), pp. 1-8.

[48] Qing, Li, Qingxin Zhu, and Mingwen Wang. "Design of a distributed energy-efficient clustering algorithm for heterogeneous wireless sensor networks." Computer communications 29, no. 12: 2230-2237. 2006.

[49] Yoon, Sunhee, and Cyrus Shahabi. "The Clustered AGgregation (CAG) technique leveraging spatial and temporal correlations in wireless sensor networks." ACM Transactions on Sensor Networks (TOSN) 3, no. 1: 3. 2007.

[50] Abbasi, Ameer Ahmed, and Mohamed Younis. "A survey on clustering algorithms for wireless sensor networks." Computer communications 30, no. 14-15: 2826-2841. 2007.

[51] Li, Xinlu, Brian Keegan, Fredrick Mtenzi, Thomas Weise, and Ming Tan. "Energy-Efficient Load Balancing Ant Based Routing Algorithm for Wireless Sensor Networks." IEEE Access 7: 113182-113196. 2019.

[52] Ch. Rami Reddy, K. Harinadha Reddy, and K. Venkata Siva Reddy. "Recognition of islanding data for multiple distributed generation systems with ROCOF shore up analysis." Smart Intelligent Computing and Applications. Springer, Singapore,. 547-558. 2019.

[53] Fouad, Mohamed Mostafa, Nour E. Oweis, Tarek Gaber, Maamoun Ahmed, and Vaclav Snasel. "Data mining and fusion techniques for WSNs as a source of the big data." Procedia Computer Science 65: 778-786. 2015.

[54] V. Krishna Chaithanya, A. Pandian, RBR Prakash, Ch. Rami Reddy"Analysis of Closed Loop control of Cascaded Three Phase Grid Tied Inverter using Fuzzy Logic Controller", International Journal of Advanced Trends in Computer Science and Engineering, Volume 8, No.4, July - August 2019.

[55] Nayyar, Anand, and Rajeshwar Singh. "A comprehensive review of ant colony optimization (ACO) based energyefficient routing protocols for wireless sensor networks." International Journal of Wireless Networks and Broadband Technologies (IJWNBT) 3, no. 3: 33-55. 2014.

[56] HUANG, Ru, Peng MIAO, and Zhihua CHEN. "The Prediction-Mode Routing Mechanism Based on ACO in WSN [J]." Chinese Journal of Sensors and Actuators 5 (2010).

[57] Jiang, Xuepeng, and Bei Hong. "ACO based energy-balance routing algorithm for WSNs." In International Conference in Swarm Intelligence, pp. 298-305. Springer, Berlin, Heidelberg, 2010.

[58] Kumar, Sanjay, Mayank Dave, and Surender Dahiya. "ACO based QoS aware routing for wireless sensor networks with heterogeneous nodes." In Emerging Trends in Computing and Communication, pp. 157-168. Springer, New Delhi, 2014.

[59] Li, Xinlu, Brian Keegan, Fredrick Mtenzi, Thomas Weise, and Ming Tan. "Energy-Efficient Load Balancing Ant Based Routing Algorithm for Wireless Sensor Networks." IEEE Access 7: 113182-113196. 2019.

[60] Ch. Rami Reddy and K. Harinadha Reddy "Passive Islanding Detection Technique for Integrated Distributed Generation at Zero Power Balanced Islanding”, International Journal of Integrated Engineering, 11(6): September 2019. 
[61] Sun, Yongiun, Wenxin Dong, and Yahuan Chen. "An improved routing algorithm based on ant colony optimization in wireless sensor networks." IEEE communications Letters 21, no. 6: 1317-1320. 2017.

[62] Collotta, Mario, Lucia Lo Bello, and Giovanni Pau. "A novel approach for dynamic traffic lights management based on Wireless Sensor Networks and multiple fuzzy logic controllers." Expert Systems with Applications 42, no. 13: 5403-5415. 2015.

[63] Gaijar, Sachin, Mohanchur Sarkar, and Kankar Dasgupta. "FAMACROW: Fuzzy and ant colony optimization based combined mac, routing, and unequal clustering cross-layer protocol for wireless sensor networks." Applied Soft Computing 43 (2016): 235-247.

[64] Reddy, KV Siva, et al. "Resonance Propagation and Elimination in Integrated and Islanded Micro grids." International Journal of Power Electronics and Drive Systems 9.3 (2018): 1445.

[65] Sun, Enyan, Chuanyun Wang, and Feng Tian. "A survey on clustering routing protocols based on PSO in WSN." TELKOMNIKA Indonesian Journal of Electrical Engineering 12, no. 7 (2014).

[66] Wang, Jin, Chunwei Ju, Yu Gao, Arun Kumar Sangaiah, and Gwang-jun Kim. "A PSO based energy efficient coverage control algorithm for wireless sensor networks." Comput. Mater. Contin 56: 433-446. 2018.

[67] Banka, Haider, and Prasanta K. Jana. "PSO-based multiple-sink placement algorithm for protracting the lifetime of wireless sensor networks." In Proceedings of the second international conference on computer and communication technologies, pp. 605-616. Springer, New Delhi, 2016.

[68] Alomari, Abdullah, William Phillips, Nauman Aslam, and Frank Comeau. "Swarm intelligence optimization techniques for obstacle-avoidance mobility-assisted localization in wireless sensor networks." IEEE Access 6: 22368-22385. 2018.

[69] Chang, Yuchao, Hongying Tang, Baoqing Li, and Xiaobing Yuan. "Distributed joint optimization routing algorithm based on the analytic hierarchy process for wireless sensor networks." IEEE Communications Letters 21, no. 12: 2718-2721. 2017.

[70] Min, Wang, and Li Shining. "An energy-efficient load-balanceable multipath routing algorithm based on AHP for wireless sensor networks." In 2010 IEEE International Conference on Intelligent Computing and Intelligent Systems, vol. 2, pp. 251-256. IEEE, 2010.

[71] Chang, Yuchao, Hongying Tang, Baoqing Li, and Xiaobing Yuan. "Distributed joint optimization routing algorithm based on the analytic hierarchy process for wireless sensor networks." IEEE Communications Letters 21, no. 12: 2718-2721. 2017.

[72] Ch. Rami Reddy and K. Harinadha Reddy. "Islanding detection for inverter based distributed generation with Low frequency current harmonic injection through Q controller and ROCOF analysis." Journal of Electrical Systems 14.22018.

[73] Chang, Yuchao, Xiaobing Yuan, Baoqing Li, Dusit Niyato, and Naofal Al-Dhahir. "Machine-learning-based parallel genetic algorithms for multi-objective optimization in ultra-reliable low-latency WSNs." IEEE Access 7 : 4913-4926. 2018.

[74] Suresh, K., et al. "A passive islanding detection method for hybrid distributed generation system under balanced islanding." Indonesian Journal of Electrical Engineering and Computer Science 14.1, 9-19. 2019.

[75] Peng, Ji, Lemin Li, and Shizhong Xu. "A novel energy efficient and reliable clustering algorithm in wireless sensor networks." 596-599. 2007.

[76] Khalil, Md Ibrahim, Md Abir Hossain, Md Japirul Haque, and Md Nahid Hasan. "EERC-MAC: Energy efficient Receiver Centric MAC protocol for Wireless Sensor network." In 2017 IEEE International Conference on Imaging, Vision \& Pattern Recognition (icIVPR), pp. 1-5. IEEE, 2017.

[77] ZHAGN, Qing, De-xiang QU, and Qiao-lin CHAI. "Energy-Efficient Reliable Clustering Algorithm Based on Complete Graph [J]." Computer Engineering 5 (2010).

[78] Feng, Sen, Liangrui Tang, and Jianhong Hao. "An energy efficient routing algorithm based on radar chart for wireless sensor networks." In 2014 11th International Conference on Fuzzy Systems and Knowledge Discovery (FSKD), pp. 840-844. IEEE, 2014.

[79] Ch. Rami Reddy and K. Harinadha Reddy. "An efficient passive islanding detection method for integrated DG system with zero NDZ." International Journal of Renewable Energy Research 8.4: 1994-2002. 2018.

[80] Fu, Chunyao, Zhifang Jiang, W. E. I. Wei, and Ang Wei. "An energy balanced algorithm of LEACH protocol in WSN." International Journal of Computer Science Issues (IJCSI) 10, no. 1: 354. 2013.

[81] Arumugam, Gopi Saminathan, and Thirumurugan Ponnuchamy. "EE-LEACH: development of energy-efficient LEACH Protocol for data gathering in WSN." EURASIP Journal on Wireless Communications and Networking 2015, no. 1: 76. 2015.

[82] Dewli, Neeraj, and R. Saini. "A Comparative Analysis of M-GEAR and MODLEACH Energy Efficient WSN Protocols." International Journal of Computer Science and Information Technologies (IJCSIT) 6, no. 3: 2641-2644. 2015.

[83] Ch. Rami Reddy, and K. Harinadha Reddy. "Islanding Detection Techniques for Grid Integrated DG-A Review." International Journal of Renewable Energy Research 9.2: 960-977. 2019.

[84] Jibreel, F. "Improved-Gateway-Based Energy-Aware Multi-Hop Routing Protocol for WSNs." International Journal of Innovative Science and Research Technology 3, no. 12: 625-630. 2018.

[85] Swarnalatha, T., T. Anuja, BV Ramana Reddy, and Ch Rami Reddy. "Spatial Data Warehousing for Integrated Urban Data Management. 2019.

[86] "Kumar, Gulshan, and Mritunjay Kumar Rai."An energy efficient and optimized load balanced localization method using CDS with one-hop neighbourhood and genetic algorithm in WSNs." Journal of Network and Computer Applications 78: 73-82. 2017.

[87] Mittal, Nitin, Urvinder Singh, and Balwinder Singh Sohi. "An energy-aware cluster-based stable protocol for wireless sensor networks." Neural Computing and Applications 31, no. 11: 7269-7286. 2019. 